\title{
Mechanical Characterization of \\ Nanocelluloses/Cellulose Acetate Composite Nanofibrous Membranes
}

\author{
Nasrin Attari ( $\square$ nasrin.attari.1@ens.etsmtl.ca ) \\ École de technologie supérieure https://orcid.org/0000-0003-1329-8224 \\ Robert Hausler \\ École de technologie supérieure
}

\section{Research Article}

Keywords: Nanocelluloses, cellulose acetate, composite electrospun nanofibers, mechanical properties, heat post treatment, TEMPO-Oxidized cellulose nanofibril, cellulose nanocrystal

Posted Date: January 20th, 2022

DOI: https://doi.org/10.21203/rs.3.rs-1214943/v1

License: (c) (i) This work is licensed under a Creative Commons Attribution 4.0 International License.

Read Full License 


\title{
Mechanical characterization of nanocelluloses/cellulose acetate composite nanofibrous membranes
}

\author{
Nasrin Attari • Robert Hausler
}

Received: date / Accepted: date

\begin{abstract}
Extensive research on membrane technologies stems from the necessity for drinking water purification, wastewater treatment, and recycling polluted water by filtration. For the successful commercialization of a filtration process, the improvement of the mechanical properties of membrane systems is an essential factor. Pressure-driven membrane filtration processes require sufficient mechanical strength to withstand operational conditions. In this study, cellulose acetate composite nanofibrous membranes, including nanocelluloses as reinforcing additives, were synthesized by the electrospinning technique. The polymer solutions were prepared from different weight percentages of Cellulose nanocrystals (CNCs) and 2,2,6,6-tetramethyl-1-piperidinyloxyl(TEMPO) oxidized Cellulose nanofibrils (TOCNFs) from 0 to 1 wt.\% and 15 wt\% of Cellulose Acetate (CA). The impacts of heat post treatment process on the mechanical properties of composite electrospun nanofibrous membranes (ENMs) has been studied. The chemical and physical properties of the composite ENMs were investigated. The morphological structure of ultimate composite ENMs was studied by scanning electron microscopy (SEM), and the chemical interactions were demonstrated by Fourier transform infrared spectroscopy. A tensile test was performed to evaluate the mechanical properties of the composite ENMs. The highest tensile strength was achieved for heat-treated $0.25 \mathrm{TOCNF} / \mathrm{CA}$ composite nanofibrous membrane. These results verify that modifying morphology and improving mechanical strength expand
\end{abstract}

N. Attari

Civil and Environmnetal Engineering Department, La Station Expérimentale des Procédés Pilotes en Environnement (STEPPE-ÉTS), École de technologie supérieure, Montreal, Canada

Tel.: +1(438)928-7577

E-mail: nasrin.attari.1@ens.etsmtl.ca

R. Hausler

Civil and Environmnetal Engineering Department, La Station Expérimentale des Procédés Pilotes en Environnement (STEPPE-ÉTS), École de technologie supérieure, Montreal, Canada 
the application of electrospun nanofibrous membranes in water purification processes.

Keywords Nanocelluloses - cellulose acetate · composite electrospun nanofibers $\cdot$ mechanical properties $\cdot$ heat post treatment $\cdot$ TEMPO-Oxidized cellulose nanofibril $\cdot$ cellulose nanocrystal

\section{$1 \quad 1$ Introduction}

2 Cellulose acetate $(\mathrm{CA})$ is one of the most abundant polymer resources, an ester derivative of cellulose, and an essential ingredient of green plant cell walls. The most common type of CA has an acetate group on two of its every three hydroxyls (Fischer et al., 2008; Salama et al., 2018; Jiang et al., 2020; Lee et al., 2018). CA is one of the most favorable biodegradable polymers for the preparation of polymeric membranes by electrospinning which have been utilized widely in filtration-based processes in particular water purification Voisin et al. (2017); Goetz et al. (2018), wastewater treatment Del Río De Vicente (2021); Abdullah et al. (2019), biosensors Vaidya and Wilkins (1994), blood purification Janeca et al. (2021), and tissue engineering Stamatialis et al. (2008). This popularity originates from extensive bio-compatibility, biodegradability Wsoo et al. (2020), nontoxic nature, good chemical, thermal Etemadi et al. (2016), and mechanical properties and relatively low cost Aboamera et al. (2019).

Over the last decade, along with the development of nanotechnology at the forefront of research, electrospinning has become a prominent technique to synthesize polymeric nanofibrous membrane Liao et al. (2018); Aboamera et al. (2019). Electrospinning is a competitive candidate among other conventional phase inversion methods of polymeric membrane fabrication due to its low cost, ease of process, and high rate of production Liu and Hsieh (2002); Lalia et al. (2013). Nanofiber membrane production by this method is based on electrostatic force between spinneret and collector to stretch polymeric fibers from a solution. Polymer solution is ejected from syringe to a spinneret and by applying high voltage power between spinneret tip as positive electrode and fiber collector as negative electrode, polymer solution is stretched under high electrostatic force. The nanofibers are deposited on the collector when the applied electrostatic force overcomes the surface tension of solution drop Pan et al. (2019); Tijing et al. (2017); Selatile et al. (2018).

Due to the fundamental effect of electrical conductivity on electrospinning efficiency, CA with an excellent electrical conductivity is an ideal polymer that can be easily synthesized via electrospinning Wsoo et al. (2020). In comparison to many CA membranes that are produced by other conventional polymeric membrane fabrication techniques such as phase inversion method, CA electrospun nanofibrous membranes (ENMs) have high specific surface area, uniform morphology, high interconnected porosity which results in high permeability flux Zhou et al. (2016), controllable thickness, high alignment of nanofibers Aruchamy et al. (2018), and good mechanical strength Suja et al. (2017); Lee et al. (2018). 

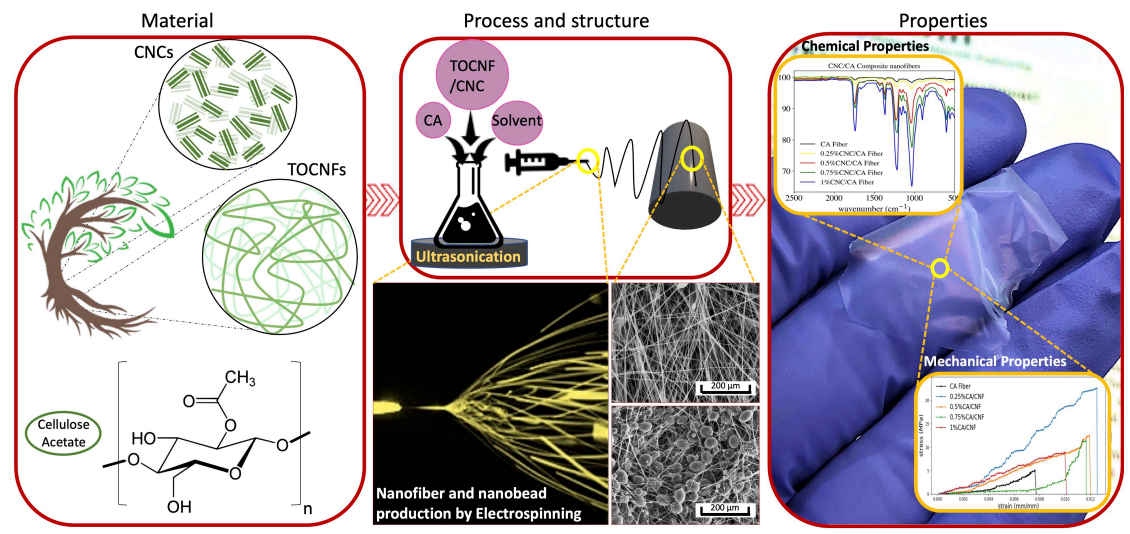

Fig. 1: Schematic representation of the chain of material-process-structureproperty in electrospun nanofibrous composite membranes (ENMs) synthesized in this study. (left) Raw CA polymer; (middle) processing of the raw material and microstructure of obtained membrane films; (right) A tiny membrane film and the obtained representative properties.

The performance of pressure driven membrane-based water purification processes from water flux and selectivity points of view depends significantly on mechanical characteristics of the membrane. According to the study of Golizadeh et al. (2019) Golizadeh et al. (2019) on mechanical properties of CA composite ENMs, tensile strength and Young's modulus of CA ENMs were approximately $1.56 \pm 0.19 \mathrm{MPa}$ and $41.6 \pm 12.3 \mathrm{MPa}$, respectively. The number of fiber cross-links per fiber length is the prominent factor that affects highly the tensile strength and Young's modulus of CA ENMs Liu and Tang (2007). The introduction of nanofillers like cellulose nanocrystals (CNCs), and cellulose nanofibrils (CNFs) to CA polymer solution and performing heat post treatment (HPT) process can improve these linkages between fibers and result in the improvement of mechanical properties Wsoo et al. (2020); Jiang et al. (2020).

CNCs, and CNFs are renewable nanoparticles originated from cellulose and depending on the source and preparation process, their lengths may vary between 100 and $2000 \mathrm{~nm}$, and diameters ranging between 2 and $20 \mathrm{~nm}$. Pretreatment step in their preparation process which highly affects their characteristics, can be chemical-based or enzymatic Mokhena et al. (2018); Goetz et al. (2018); Ma et al. (2014). The most important pretreatments are TEMPOmediated oxidation, mechanical refining, hydrolysation, and carboxymethylation. The key point in 2,2,6,6-tetramethyl-1-piperidinyloxyl (TEMPO)-mediated oxidation is the negatively charged surface of the chrystals and the fibrils. The individual fiber and chrystal due to electrostatic repulsion forces can be stayed free and results in an improved dispersion Patiño-Masó et al. (2019); Levanic et al. (2020). These nanoparticles have attracted the researchers' in- 
terest to substitute other reinforcements in composite membranes Jonoobi et al. (2010). The introduction of these nanofillers to polymeric solution is an effective technique to produce a mechanically-strong composite nanofibrous membranes Voisin et al. (2017). Furthermore, in order to cross-link the CNCs and TEMPO oxidized cellulose nanofibrils (TOCNFs) and improve the fibrous linkages, HPT process is recommended. Recently, CNFs have been successfully used as a reinforcing agent for various polymers such as Poly (lactic acid) Ghasemi et al. (2018); Jin et al. (2020), poly (vinyl alcohol) Abe et al. (2020), and poly(butylene succinate) Wang et al. (2020). In addition, CNCs have been used as nanofillers for reinforcing of poly-(e-caprolactone) (PCL) Sheng et al. (2014); Hivechi et al. (2020), poly-(acrylic acid) (PAA) Yang et al. (2012), polyethylene oxide (PEO) Mokhena et al. (2018), poly(vinyl alcohol) (PVA) Geng et al. (2020), poly(methyl methacrylate) (PMMA) Ni et al. (2019), and polyvinylidenefluoride-co-hexafluoropropylene (PVDF-HFP) Wang et al. (2019); Lalia et al. (2014). According to the pre-mentioned studies, mechanical strength of various polymeric composite nanofibrous membranes by addition of CNCs and CNFs are obviously improved.

HPT is one of the most efficient approaches to improve the mechanical strength and overcome the difficulty of ENMs' application in filtration processes Choi et al. (2004); Arribas et al. (2014); You et al. (2006). The main effectiveness of this method is enhancing the bonding between fiber linkages that results in increasing of structural integrity and mechanical strength of the membranes which facilitate the handling of ENMs for filtration processes Arribas et al. (2019); Liang et al. (2013).

In this study, CNC/CA and TOCNF/CA composite ENMs were rationally synthesized. First, pristine CA nanofibers were fabricated by the electrospinning method. Then, CNC and TOCNF reinforcing nanofillers were added to spinning polymer solutions, and composite nanofibers were synthesized. CNC and TOCNF nanoparticles were loaded to improve the mechanical characteristics of the nanofibrous membranes. The microstructure, morphologies, physical properties, chemical and mechanical properties of the samples were investigated. The effects of adding CNCs and TOCNFs with variable weight percentages $(0,0.25,0.5,0.75$, and 1 wt. $\%)$ into $\mathrm{CA}$ polymer solution on mechanical properties of nanofibrous membranes were illustrated. Furthermore, we investigated the impact of HPT process on the properties. This work provides a material-process-structure-property mapping for CA-based nanofibrous composite membranes. This map is schematically shown in Fig. 1 where at the most general level, we consider a main polymer matrix, nanofillers and the solvent. The chemical formula of CA as the main polymer matrix is shown in the left block. In the process level, we dissolved the polymer solution using ultrasonication technique until the nanofillers were well dispersed and well distributed in the spinning solution. On the right block of the Fig. 1, a synthesized ENM film is shown. The obtained samples were further characterized to determine the structure, chemical properties, and mechanical properties using scanning electron microscopy (SEM), Fourier transform infrared spectroscopy (FTIR), and universal tensile testing, respectively. 
This paper is structured as follows: The polymer materials and the details 111 of methods for the preparation of composite polymer solution and synthesis 2 of composite ENMs and HPT process are explained in section 2. In section 3, the effects of nanofiller concentration, and HPT process on the morphological structure and mechanical properties of ENMs are discussed. In this section 3, we discuss the results of FTIR and tensile strength tests to find out the improvement of mechanical properties of ENMs. Furthermore, we present a summary of our findings and draw our conclusions in section 4.

\section{Methods}

\subsection{Materials}

Cellulose Acetate $(\mathrm{CA}, \mathrm{Mn}=50000, \rho=1.3 \mathrm{~g} / \mathrm{mL}$ ), received from Sigma-Aldrich Chemistry, was used as the matrix polymer. Freeze-dried powder TEMPO oxidized cellulose nanofibrils (TOCNFs, 98 wt\% dry powder) and freeze dried powder cellulose nanocrystals (CNCs, 98 wt.\% dry powder), purchased from Cellulose LAB, Canada were used as nanofillers in the preparation of electrospun composite nanofibers. Different volume ratios of N-dimethylformamide, DMF, and acetone, purchased from Fisher Chemical Co., were used as the solvent without further purification.

\subsection{Optimization of the material and electrospinning process parameters}

In our previous work Attari and Hausler (2020), the polymer solutions of various compositions of the solvent and CA concentrations were prepared. Their morphological structure were studied to determine the optimized material and electrospinning process parameters. For the solvent composition, three volume ratios of DMF and acetone, (2:8), (1:1), and (1:0) were intended. In the case of volume ratio of $(2: 8)$, due to high volatility of acetone, the needle tip was blocked during the electrospinning. For the volume ratio of (1:0), pure DMF, the high surface tension of this solvent resulted the beads during the electrospinning process. According to the morphological results, by increasing the CA concentration from $10 \mathrm{wt} \%$ to $15 \mathrm{wt} \%$, the bead formation was declined due to the increase in solution viscosity and surface tension. On the other hand, the further increase in CA concentration to $20 \mathrm{wt} \%$ increased the viscosity of the solution and made nanofiber formation more difficult. Therefore, the CA concentration of $15 \mathrm{wt} \%$ and (1:1) solvent ratio of DMF and acetone were determined to prepare polymer solutions for this study. The efficient values for process parameters were determined in regard to morphological study in our previous work. The electrospinning process parameters values such as voltage, polymer solution feed rate, tip to collector distance (TCD), and process time were kept constant at $25 \mathrm{kV}, 2 \mathrm{ml} / \mathrm{h}, 100 \mathrm{~mm}$, and $2 \mathrm{~h}$, respectively. 
2.3 Preparation of $\mathrm{CNC} / \mathrm{CA}$ and $\mathrm{TOCNF} / \mathrm{CA}$ composite polymer solutions

The polymer solutions were prepared by dispersing $15 \mathrm{wt} \%$ of $\mathrm{CA}$ and different concentrations of CNC or TOCNF nanofillers in a mixture solvent of DMF and acetone (volume ratio of $1: 1$ ) as follows. The calculated amount of CA was stirred in half volume of solvent for $24 h$ using magnetic stirrer at 300 rpm at ambient temperature until the polymer was homogeneously dissolved. Using a high speed ultrasonic disperser, the determined amount of CNCs or TOCNFs in regard to the varied weight percentage of the nanofillers from $0 \mathrm{wt} \%$ to $1 \mathrm{wt} \%$ were dispersed in other half volume of solvent at 30,000 rpm until homogeneous suspension obtained. Finally, the two solutions were ultrasonicated until homogeneous dispersion of nanofiller in spinning solution obtained. Table 1 presents the detailed sample codes, the values of nanofiller concentration, and the implementation of heat post treatment (HPT) in the synthesize process.

Table 1: The composition and formulation of prepared spinning solutions

\begin{tabular}{lllll}
\hline Sample Code & $\begin{array}{l}\text { CA } \\
\text { (wt.\%) }\end{array}$ & $\begin{array}{l}\text { CNC } \\
(\text { wt. } \%)\end{array}$ & $\begin{array}{l}\text { TOCNF HPT } \\
\text { (wt.\%) }\end{array}$ \\
\hline CA & 15 & 0 & & $\times$ \\
CA-HPT & 15 & 0 & & $\checkmark$ \\
$0.25-C N C-C A$ & 15 & 0.25 & & $\times$ \\
$0.25-C N C-C A-H P T$ & 15 & 0.25 & & $\checkmark$ \\
$0.5-C N C-C A$ & 15 & 0.5 & & $\times$ \\
$0.5-C N C-C A-H P T$ & 15 & 0.5 & & $\checkmark$ \\
$0.75-C N C-C A$ & 15 & 0.75 & & $\times$ \\
$0.75-C N C-C A-H P T$ & 15 & 0.75 & & $\checkmark$ \\
1-CNC-CA & 15 & 1 & & $\times$ \\
1-CNC-CA-HPT & 15 & 1 & & $\checkmark$ \\
$0.25-C N F-C A$ & 15 & & 0.25 & $\times$ \\
$0.25-C N F-C A-H P T$ & 15 & & 0.25 & $\checkmark$ \\
$0.5-C N F-C A$ & 15 & & 0.5 & $\times$ \\
$0.5-C N F-C A-H P T$ & 15 & & 0.5 & $\checkmark$ \\
$0.75-C N F-C A$ & 15 & & 0.75 & $\times$ \\
$0.75-C N F-C A-H P T$ & 15 & & 0.75 & $\checkmark$ \\
1-CNF-CA & 15 & & 1 & $\times$ \\
1-CNF-CA-HPT & 15 & & 1 & $\checkmark$ \\
\hline
\end{tabular}

\subsection{Electrospining process}

The preparation procedure of polymer solutions and the electrospinning process of CA nanofibers were described in the previous work Attari and Hausler (2020). The set-up consists of a $20 \mathrm{~mL}$ BD plastic syringe as the solution container which is connected to a nozzle with inner diameter of $0.8 \mathrm{~mm}$ to form the fibers. A pump controls the feed rate of the solution. The produced 
nanofiber samples are collected using a collector that is covered by aluminum foil to facilitate the peeling off the membrane from the collector. Furthermore, a power supplier $(0-40 \mathrm{kV})$ is used to make the spinneret and the collector as two electrodes to provide electrostatic force to form the nanofibrous membrane mats. CNC/CA and TOCNF/CA composite spinning solutions with 15 wt.\% $\mathrm{CA}$ and variable $\mathrm{CNC}$ and TOCNF loadings were prepared. To achieve maximum efficiency, different concentrations of CNCs and TOCNFs were added to previously obtained optimum solvent composition Attari and Hausler (2020). The composition of the optimum solvent is dissolution of $15 \mathrm{wt} . \% \mathrm{CA}$ polymer in the equal volume ratio (1:1) of $\mathrm{N}, \mathrm{N}$-dimethylformamide (DMF) and acetone mixture solvent. All CNC/CA and TOCNF/CA ENMs were synthesized under previously obtained optimum electrospinning process parameter conditions: a polymeric solution flow rate of $2 \mathrm{~mL} / \mathrm{h}$, a distance between spinneret tip and collector (TCD) of $100 \mathrm{~mm}$, an electric voltage of $25 \mathrm{kV}$ and an electrospinning process time of $2 h$ Attari and Hausler (2020). The electrospinning process was performed in temperature range from $20{ }^{\circ} \mathrm{C}$ to $26{ }^{\circ} \mathrm{C}$ and humidity range from $30 \%$ to $51 \%$.

\subsection{Heat Post Treatment of CNC/CA, TOCNF/CA composite nanofibers}

The loose and fluffy texture of the ENMs make it difficult to handle them after the fabrication step. HPT process was conducted to improve the coherence structure and mechanical resistance of the CNC/CA and TOCNF/CA ENMs. HPT was carried out in a glass beaker that contained deionized (DI) water in $70^{\circ} \mathrm{C}$ which must be higher than the boiling point of the solvent mixture in order to complete evaporate of the solvent from the synthesized ENMs and lower than the glass transition temperature of the $\mathrm{CA}$ matrix polymer $\left(110^{\circ} \mathrm{C}\right)$ to form good connectivity between the nanofibers right after electrospinning step for $2 \mathrm{~h}$. The nanofibrous membranes were stored at ambient temperature for $24 \mathrm{~h}$ to be dried.

\subsection{Characterization}

\subsubsection{Solution characterization}

The electrical conductivity of spinning solutions was measured using a conductivity meter (OAKLON pH/CON 510 Benchtop Meter). The conductivity electrode was entirely submerged in the solution, and at the room temperature, the conductivity measurement was carried out after stabilizing the reader. The viscosity of spinning solutions was determined by a digital viscometer (Brookfield) in a $20 \mathrm{~mL}$ cylindrical sample container at a constant solution temperature of $25^{\circ} \mathrm{C}$ using an S-31 spindle. The rotation frequency of the S-31 spindle was $50 \mathrm{rpm}$, and the shear rate was $10.2 s-1$. 
2.6.2 Morphological analysis

The morphological structure of CNC/CA and TOCNF/CA composite nanofibers was investigated by a Scanning Electron Microscope (SEM), Hitachi Model S3600-N. The SEM was performed at $5 \mathrm{kV}$ in three magnifications. The SEM samples were coated with gold using a sputter coater (Quorum Technologies Model K550X) under $35 \mathrm{~mA}$ current for 2 minutes.

The fiber distribution and mean fiber diameter size were measured using image processing software (ImageJ, 2.0.0-rc-43/1.50e) based on 40 fibers.

\subsubsection{Mechanical testing}

Mechanical properties of samples were measured on a Pneumatic tensile machine (Alliance RF/200(MTS)) at a loading speed of $2 \mathrm{~mm} / \mathrm{min}$ equipped with load cell of $100 \mathrm{~N}$. All the ENM samples were measured $70 \mathrm{~mm}$ long by 10 $\mathrm{mm}$ wide. The upper and lower chucks were clamped to the sample at $10 \mathrm{~mm}$. An averaged value from three replicates was taken for all samples. The room temperature and relative humidity was controlled constant at $25 \circ$ and $50 \%$, respectively.

\subsubsection{Fourier transform infrared spectroscopic analysis (FTIR)}

Fourier transform infrared (FTIR, Perkin Elmer) spectroscopic analyses of all samples were done with a resolution of $2 \mathrm{~cm}^{-1}$ by averaging 64 scans in the range of 4000-400 $\mathrm{cm}^{-1}$. FTIR of all nanofibrous membranes were taken under an attenuated total reflection (ATR) mode using corresponding accessory.

\section{Results and discussion}

3.1 Effects of nanofiller addition and heat post treatment process on the structural morphologies of CA composite nanofibrous membranes

In this study, CNC/CA and TOCNF/CA solutions composed of $15 \mathrm{wt} \%$ matrix $\mathrm{CA}$ polymer and four concentrations of $0.25,0.5,0.75$, and $1 \mathrm{wt} \% \mathrm{CNC}$ or TOCNF nanofillers in 1:1 volume ratio of DMF and acetone mixture solvent were electrospun. Electrospinning method is used to prepare the composite nanofibrous membranes and study the effects of nanofiller concentration and HPT process on microstructure and mechanical properties during constant electrospinning process conditions. The following sections describe the impact of heat treatment process, and the concentration of CNCs and TOCNFs on bead free morphological structure and accordingly on mechanical properties of the CA nanofibers, respectively. 


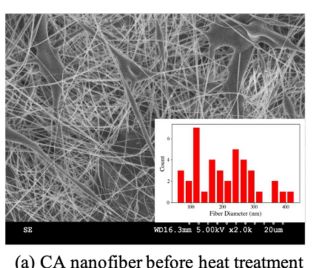

(a) CA nanofiber before heat treatment

$\mathrm{CNC} / \mathrm{CA}$ nanofiber before and after heat treatment

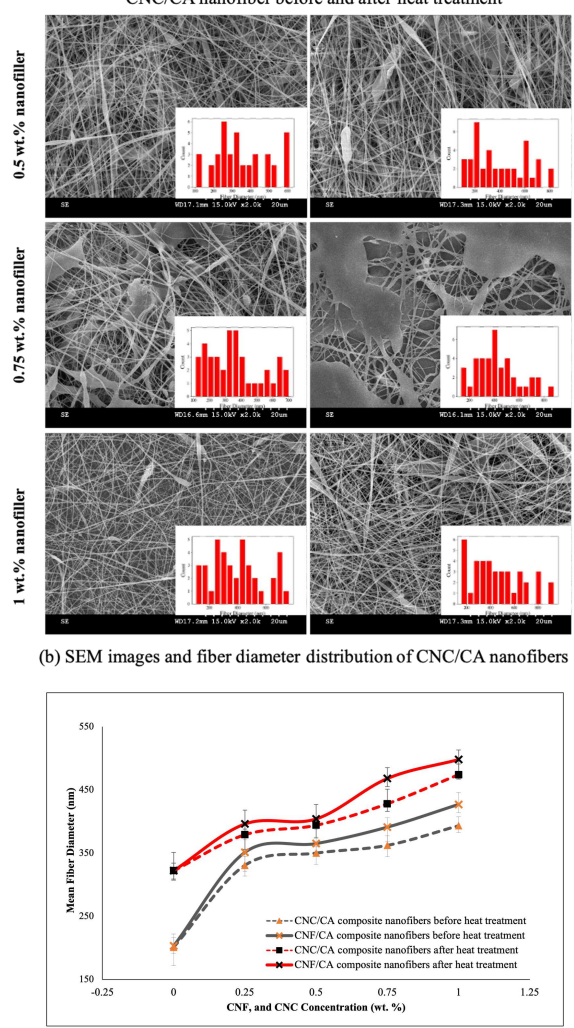

(d) Mean fiber diameter

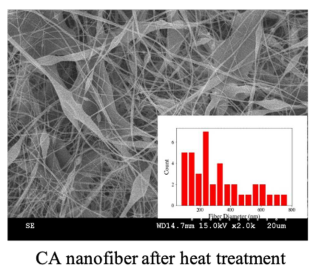

$\mathrm{CNF} / \mathrm{CA}$ nanofiber before and after heat treatment

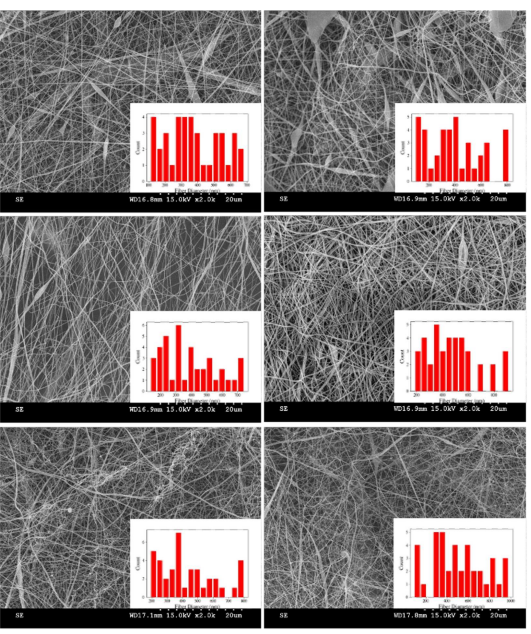

(c) SEM images and fiber diameter distribution of CNF/CA nanofibers

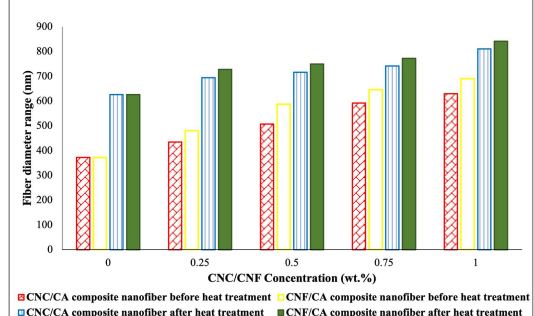

(e) Fiber diameter range

Fig. 2: SEM images, fiber diameter distribution and range for pristine CA nanofibers and $\mathrm{CNC} / \mathrm{CA}$ and TOCNF/CA composite nanofibers before and after heat post treatment process (a)Pristine CA nanofibers (b)CNC/CA composite nanofibers (c)TOCNF/CA composite nanofibers (d)Mean fiber diameter vs. nanofiller loading (e)Fiber diameter range vs. nanofiller loading

\subsubsection{Structural effects of heat post treatment process}

The SEM micrographs of pristine CA ENMs before and after heat treatment process are shown in Fig. 2 (a). The CA nanofibers were physically joined together by thermal treatment and due to slight surface melting the diameter 
of the fibers were increased. According to the Fig. 2(a), the fiber distribution is widened after heat treatment and mean fiber diameter is increased from $0.203 \mathrm{~nm}$ to $0.270 \mathrm{~nm}$.

\subsubsection{The effect of nanofiller concentration on conductivity and viscosity of} composite spinning solutions

In this section, we study the effect of concentration of CNCs and TOCNFs on the conductivity and viscosity of the composite spinning solutions. In general, the final morphology of nanofibers are highly affected by several solution parameters such as viscosity and conductivity. The conductivity and viscosity of CA composite solutions for different concentrations of CNCs and TOCNFs are summarized in Fig. 3(a) and (b), respectively. The viscosity and also the conductivity of $\mathrm{CNC} / \mathrm{CA}$ spinning solutions were increased with the elevation of CNC concentrations from 0 wt.\% to $1 \mathrm{wt} . \%$. The same increasing trend could also be seen for TOCNF/CA spinning solutions in Fig. 3(a) and (b).

At the equal concentration, the viscosity and conductivity of the TOCNF/CA solutions are higher than $\mathrm{CNC} / \mathrm{CA}$ solutions. This could be due to the fibrous molecules of TOCNFs which affected the entanglement with CA.

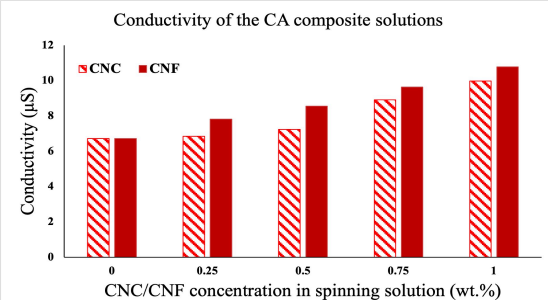

(a)

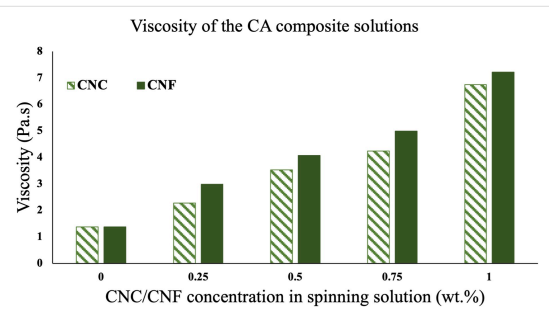

(b)

Fig. 3: The impact of CNC/TOCNF concentration on a) conductivity and b) viscosity of the spinning solutions

\subsubsection{Effect of CNC and TOCNF addition on microstructure of Composite nanofibers}

The SEM micrographs and corresponding fiber diameter distribution graphs of CNC/CA and TOCNF/CA ENMs before and after HPT process are shown in Fig. 2 (b), and (c). The various morphologies as a function of CNC and TOCNF concentration $(0.25,0.5,0.75$, and $1 \mathrm{wt} . \%)$ are demonstrated. Additionally, in Fig. 2 (b), and (c) the effect of HPT on the morphology of various $\mathrm{CNC} / \mathrm{CA}$ and TOCNF/CA composite nanofibers along with their fiber diameter distribution can be observed. As discussed for pristine CA ENMs in the previous section, the fiber diameter distributions are widened after heat post 
treatment process. Fig. 2 (d) summarizes mean fiber diameters of CNC/CA and TOCNF/CA composite nanofibers in relation to nanofiller concentration before and after HPT. As can be seen in Fig. 2 (d), for CNC/CA composite nanofibers, mean fiber diameter is increased from $203 \mathrm{~nm}$ to $393 \mathrm{~nm}$ by the augmentation of CNC concentration from 0 to $1 \mathrm{wt} . \%$ in the composite spinning solution and for TOCNF/CA composite nanofibers, an increase of mean fiber diameter from $203 \mathrm{~nm}$ to $427 \mathrm{~nm}$ is observed with augmented TOCNF concentration from 0 to $1 \mathrm{wt} . \%$ in the composite spinning solution. This observance is in correspondence with the results from Fig. 3 (a) and (b) which are described in the previous section. The increasing of CNC and TOCNF concentration result in the elevation of both viscosity and conductivity of the solutions and they require more electrostatic force to form nanofibers with the same diameter. Hence in constant spinning conditions, the increasing of viscosity results in elevation of fiber diameter. Fig. 2 (e) shows that the fiber diameter range is widened by increasing the CNC and TOCNF concentration. Wider fiber diameter distribution in TOCNF/CA composite nanofibers in comparison to $\mathrm{CNC} / \mathrm{CA}$ composite nanofibers has root in higher viscosity due to fibrous TOCNF molecules. Furthermore, according to the Fig. 2 (e) HPT process increases the fiber diameter range. According to the Fig. 2 (d), and the description of CNC/TOCNF concentration and heat post treatment effects, the highest mean fiber diameter is obtained for 1-CNF-CA-HPT sample which is composed of 15 wt.\% CA, 1 wt.\% TOCNFs, equal volume fractions of DMF and acetone as solvent and heat treatment is performed on it.

The FTIR spectrums of CNC/CA composite nanofibrous membranes and TOCNF/CA composite nanofibrous membranes for different concentrations of the nanofillers in the wavenumber range of 500-4000 $\mathrm{cm}^{-1}$ are given in Fig. 4 a, and b, respectively. The dominant spectral bands at $1031 \mathrm{~cm}^{-1}, 1218$ $\mathrm{cm}^{-1}$, and $1738 \mathrm{~cm}^{-1}$ are corresponding to the stretching vibrations of $\mathrm{C}-\mathrm{O}$ and $-\mathrm{C}=\mathrm{O}$ carboxyl groups from residual acetate groups in the $\mathrm{CA}$ matrix. The bands near $1369 \mathrm{~cm}^{-1}$ and $900 \mathrm{~cm}^{-1}$ correspond to the $\mathrm{C}-\mathrm{H}$ bending vibration of -CH3 group. The peak around $600 \mathrm{~cm}^{-1}$ is the bending vibration of $-\mathrm{CH}$. The presence of a band at $1639 \mathrm{~cm}^{-1}$ due to $-\mathrm{OH}$ bending indicates the presence of water in the structure of the nanofibers. The increased peak area is due to the increased concentration of nanofiller, which is evidence of more $-\mathrm{OH}$ vibration from adsorbed water. The TOCNF chemical structure is illustrated in Fig. 4-b. According to Fig. 4 a, and b, by increasing CNC loading, the absorption peaks became stronger gradually. However, in the case of TOCNFs, by elevation of Nanofiller concentration from $0 \mathrm{wt} \%$ to $1 \mathrm{wt} \%$ the absorption peaks are remained almost constant at the concentration of $0 \mathrm{wt} \%$ to $0.75 \mathrm{wt} \%$, and with further increase in TOCNF concentration to $1 \mathrm{wt} \%$ strong absorption peaks are obtained. The small peaks were observed at 2920 $\mathrm{cm}^{-1}$ (-CH stretching vibrations from alkyl groups) and $3420 \mathrm{~cm}^{-1}$ (free -OH stretching vibrations of -OH groups) in FTIR spectra of 1-CNF-CA composite ENM. The peak at $3420 \mathrm{~cm}^{-1}$ indicates the formation of $\mathrm{H}$-bonding between TOCNF molecule and adsorbed water. 


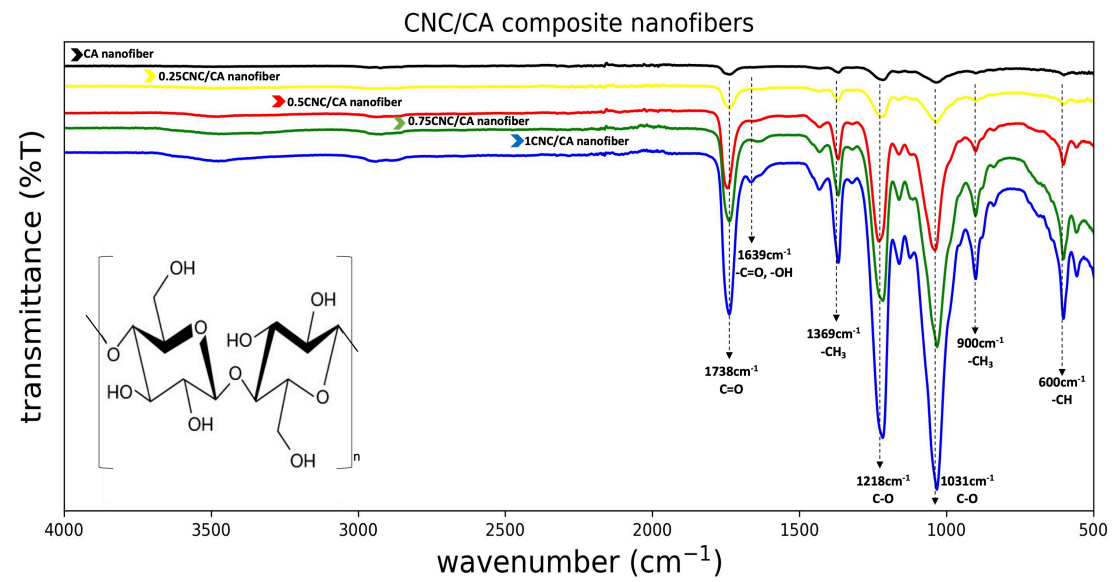

(a)

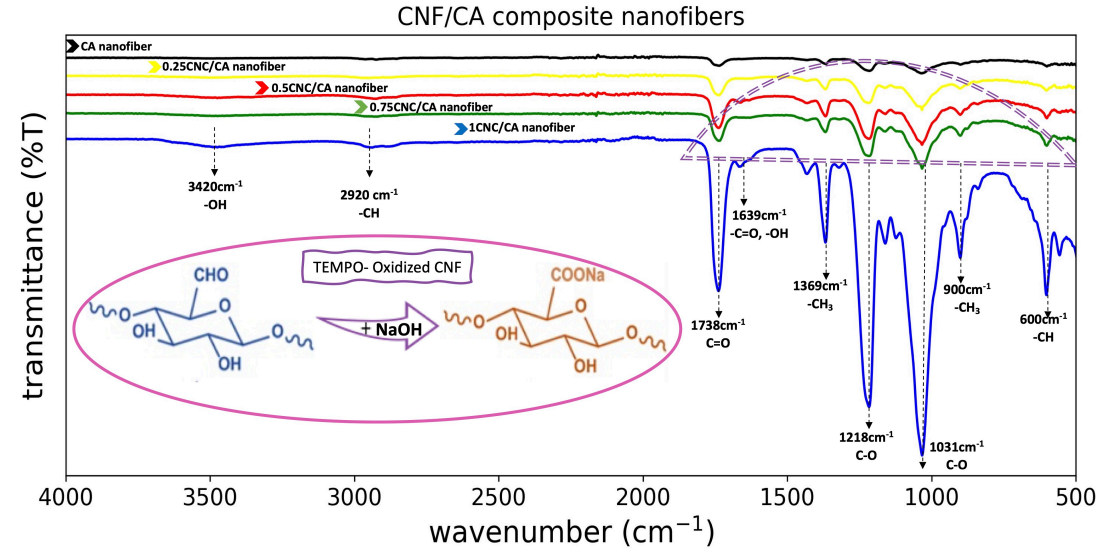

(b)

Fig. 4: FTIR spectras of ENMs (a) CNC/CA composite nanofibers (b) TOCNF/CA composite nanofibers before heat post treatment (For interpretation of the colors in this figure please refer to online version of this document.)

Fig. 5 shows the FT-IR spectra acquired from the 0.5-CNC-CA nanofibers before and after heat treatment process. All the coinciding peaks indicate that heat treatment process does not cause any compositional change of the composite nanofibers.

All these results are proofs of the feasible morphological controlling of the CA nanofibers using an efficient, effective procedure for achieving the desired surface morphology. 


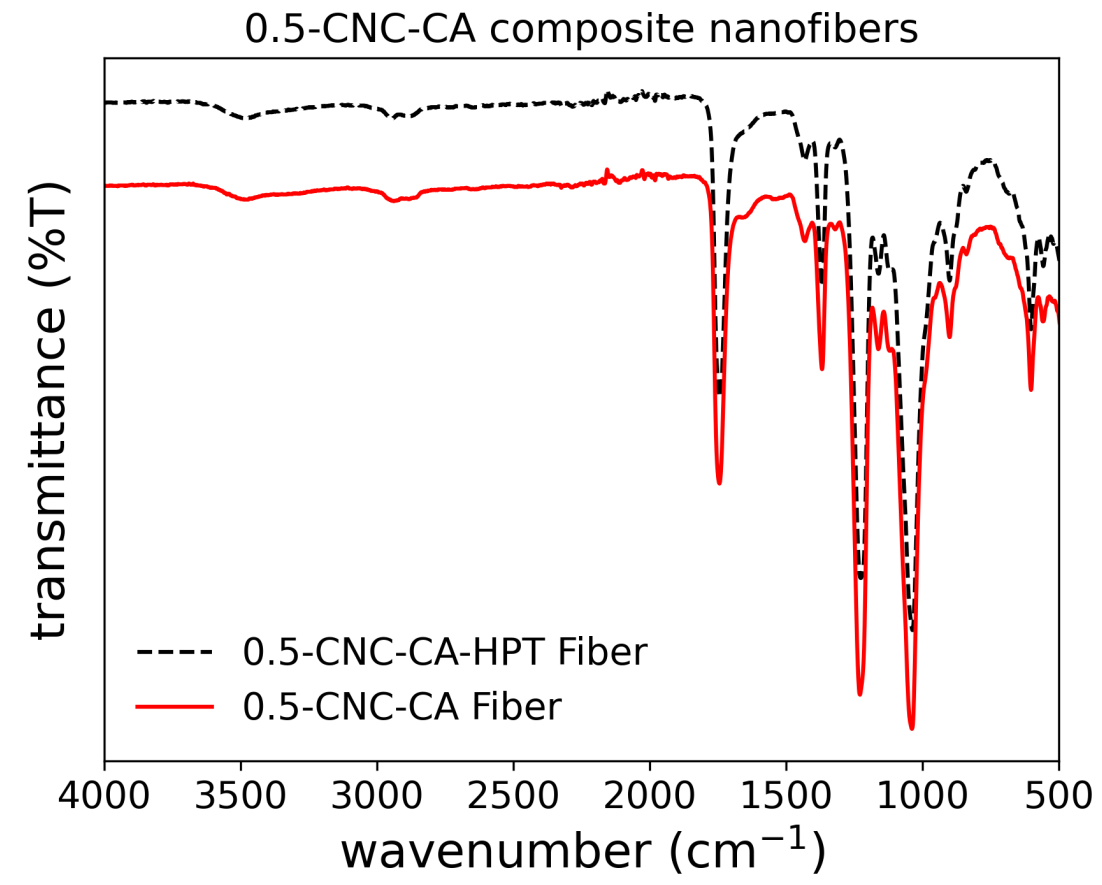

Fig. 5: FTIR spectras of 0.5-CNC-CA nanofibrous composite membranes before and after heat post treatment process

\subsection{Mechanical properties}

Fig. 6 presents stress strain curves for $\mathrm{CNC} / \mathrm{CA}$ and TOCNF/CA composite nanofibrous membranes with different reinforcement concentrations for before and after heat treatment process. The impact of the increase in concentration of CNC and TOCNF nanofillers on the fracture stress and strain is investigated in Fig. 6-a and b, respectively. The fracture stress and strain of pristine CA nanofibrous membrane is $5.099 \mathrm{MPa}$ and $0.76 \%$, respectively. By increasing the $\mathrm{CNC}$ concentration from $0 \mathrm{wt} \%$ to $1 \mathrm{wt} \%$, both fracture stress and fracture strain reach a maximum of $15.39 \mathrm{MPa}$, and $1.18 \%$ at the CNC concentration of $0.5 \mathrm{wt} \%$ which are 3.02 times and 1.55 times that of pristine CA nanofibrous membrane, respectively (Fig. 6-a). Similar works which are introduced CNCs as nanofiller to the structure of nanofibers, only showed 1.4 times (Sun et al. (2015))Sun et al. (2015) and 2.2 times (Jiang et al. (2020) Jiang et al. (2020) improvement in fracture strength of CNC/CA composite nanofibers in comparison to $\mathrm{CA}$ nanofibers. As can be seen in Fig. 6 -b, the maximum fracture stress and strain of $22.6 \mathrm{MPa}$, and $1.25 \%$ are achieved for 0.25-CNF-CA composite nanofiber membranes which are 4.43 

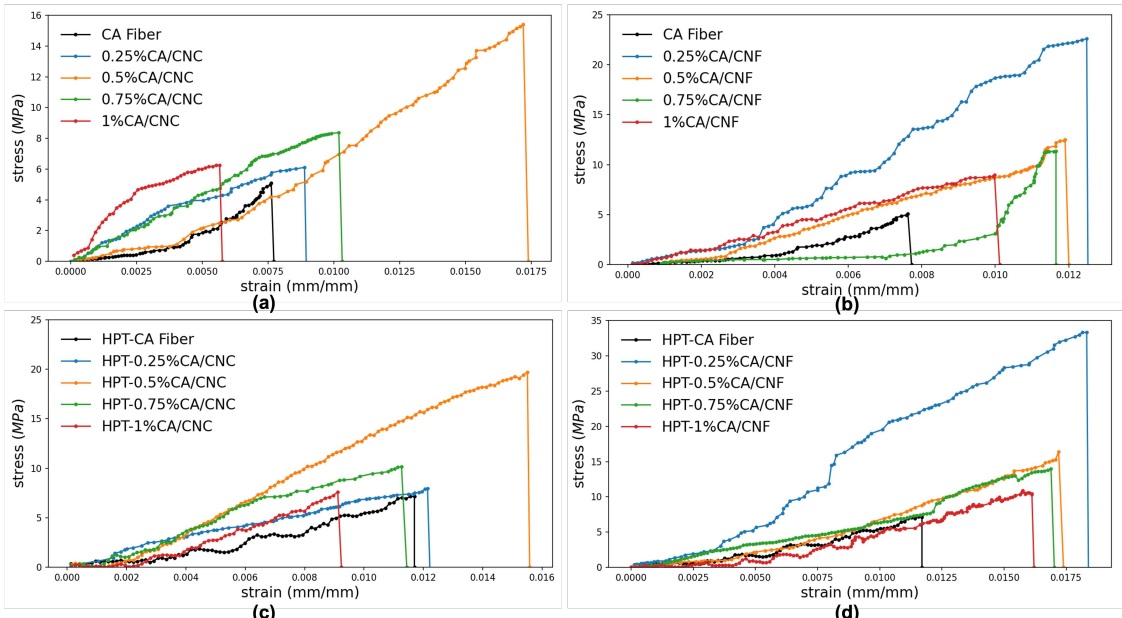

Fig. 6: The effect of (a) CNC and (b) TOCNF addition and (c),(d) Heat Post Treatment on mechanical properties of CA ENMs (For interpretation of the colors in this figure please refer to online version of this document.)

times and 1.65 times those of pristine CA nanofiber membranes, respectively. The decrease in tensile strength above $0.5 \mathrm{wt} \% \mathrm{CNCs}$ and $0.25 \mathrm{wt} \%$ TOCNFs could be due to the potential aggregation of them. According to Fig. 6-a and $\mathrm{b}$, the decrease in tensile strength due to the increase in TOCNF concentration above $0.25 \mathrm{wt} \%$ is higher than in case of CNCs. This could be due to stronger aggregation of TOCNFs and stronger hydrogen bonding than in case of CNCs. The considerable improvement in fracture strength of composite nanofibrous membranes is due to the reinforcement effect of CNCs and TOCNFs. The higher tensile strength properties of nanocomposites containing TOCNFs than those containing CNCs could be attributed to the stronger ionic interaction between the negatively charged TOCNFs and the positively charged CA chains.Additionally, it may has roots in the fibrous structure of TOCNFs which results in higher interaction and cross linking with CA matrix. The main reason of the serious decline in mechanical properties of the composite nanofibers by increasing the CNC and TOCNF concentration, may be related to the formation of aggregates in higher concentrations. The uniform distribution and dispersion of nanofillers in the structure of nanofibers play a prominent role in their mechanical properties. Figures 6-c, and d demonstrate the effect of heat treatment process on mechanical properties of the $\mathrm{CNC} / \mathrm{CA}$, and TOCNF/CA composite nanofibers. The improvement in both fracture stress, and fracture strain was mainly due to cross linking between the nanofibers after heat treatment led to bonding between nanofibers at the crossover points.In addition, solvents removal from the nanofibers due to heat treatment can improve the integrity of the membranes. As can be seen from Figures 6-c, and d, the declining rate in mechanical properties of $\mathrm{CNC} / \mathrm{CA}$, 
and TOCNF/CA composite nanofibers after heat treatment by increasing the reinforcement concentration is similar to the Figures 6-a, and b.

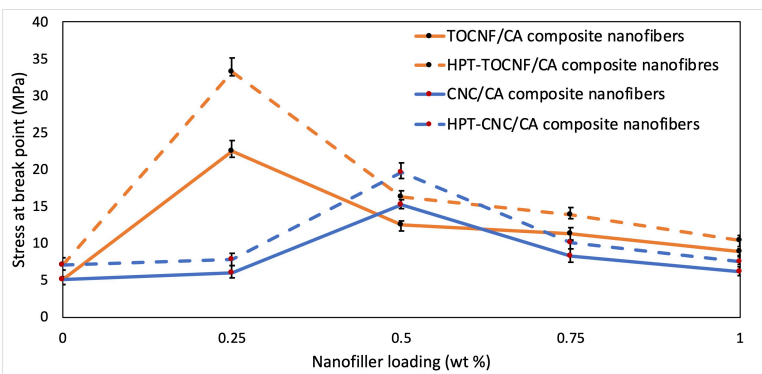

Fig. 7: Variations of stress at break points of $\mathrm{CNC} / \mathrm{CA}$ and TOCNF/CA composite nanofibrous membranes as a function of nanofiller loading and heat post treatment process (For interpretation of the colors in this figure please refer to online version of this document.)

Fig. 7 shows the comparisons of fracture strength among pristine CA nanofibers, CNC nanofiller reinforced CA composite ENMs and TOCNF nanofiller reinforced CA composite ENMs as a function of nanofiller loadings and HPT process. Overall, the addition of $\mathrm{CNC}$ and TOCNF reinforcements to CA matrix result in the elevation of fracture stress. The maximum fracture stress was occurred in heat treated $0.25 \mathrm{TOCNF} / \mathrm{CA}$ composite ENM. The addition of TOCNFs improves the flexibility of TOCNF/CA composite ENM film by the formation of a good interfacial bonding between CA matrix and the fibrils Liu et al. (2013); Wu et al. (2019). As can be seen in Fig. 7, by further increasing of TOCNF weight percentage from $0.25 \mathrm{wt} \%$ to $1 \mathrm{wt} \%$ in the polymer spinning solution the fracture strength is decreased. This decrease tendency might be caused by the inhomogeneous distribution of TOCNF nanofiller in the CA polymer matrix, caused by the high aspect ratio of TOCNFs and the formation of aggregations occurred by strong hydrogen bonds and swollen clusters by water in the solution.

\section{Conclusion}

Various CA ENMs were prepared at constant optimized CA concentration of $15 \mathrm{wt} \%$ and electrospinning parameters of polymer flow rate, $\mathrm{F}=2 \mathrm{~mL} / \mathrm{h}$, tip to collector distance, $\mathrm{TCD}=100 \mathrm{~mm}$, electric voltage, $\mathrm{V}=25 \mathrm{kV}$, and electrospinning process time, $\mathrm{t}=2 \mathrm{~h}$. By varying $\mathrm{CNC}$, and TOCNF concentration from $0 \mathrm{wt} \%$ to $1 \mathrm{wt} \%$ which were used as the reinforcements, the mechanical strength of the $\mathrm{CA}$ nanofibers was improved due to their reinforcing effects. The resulted CNC/CA and TOCNF/CA composite ENMs showed 3.02 times and 4.43 times more strength fracture than pristine CA ENMs. TOCNF reinforcement addition to the CA polymer matrix showed better reinforcing 
effects than CNC reinforcement due to its fibrous structure which results in higher interaction and cross linking with CA matrix. Furthermore, it could be attributed to the stronger ionic interaction between the negatively charged TOCNF molecules and the positively charged CA chains.

The nanofibers were physically joined together by applying the heat treatment process to the prepared ENMs and due to slight surface melting, the diameter of the fibers were increased and as a result mechanical strength of the thermal treated samples were improved.

\section{Acknowledgment}

Nasrin Attari is thankfull to Arbour Foundation, Molson Foundation, and École de technology supérieure for their financial support. Part of this research has been carried out in the STEPPE and material characterization Laboratories of ETS University. Also, NA gratefully acknowledges the support from Dr. Martha Cerruti, the head of the Biointerface Laboratory of McGill University for providing electrospinning equipment to carry out the research reported in this article.

\section{Statements and Declarations}

\section{Funding}

NA and RH thank the support by the École de Technologie Supérieure.

\section{Competing Interests}

The authors have no relevant financial or non-financial interests to disclose.

\section{Author Contributions}

NA designed, planned, performed, and processed the membrane sample preparation, synthesis, and characterization and interpreted the results. NA prepared the manuscript and RH supervised the project. The author(s) read and approved the final manuscript.

\section{References}

Abdullah N, Yusof N, Lau W, Jaafar J, Ismail A (2019) Recent trends of heavy metal removal from water/wastewater by membrane technologies. Journal of Industrial and Engineering Chemistry 76:17-38 
Abe K, Tomobe Y, Yano H (2020) The reinforcement effect of cellulose nanofiber on young's modulus of polyvinyl alcohol gel produced through the freeze/thaw method. Journal of Polymer Research 27(8):1-5

Aboamera NM, Mohamed A, Salama A, Osman T, Khattab A (2019) Characterization and mechanical properties of electrospun cellulose acetate/graphene oxide composite nanofibers. Mechanics of Advanced Materials and Structures 26(9):765-769

Arribas P, Khayet M, García-Payo M, Gil L (2014) Self-sustained electrospun polysulfone nano-fibrous membranes and their surface modification by interfacial polymerization for micro-and ultra-filtration. Separation and Purification Technology 138:118-129

Arribas P, García-Payo M, Khayet M, Gil L (2019) Heat-treated optimized polysulfone electrospun nanofibrous membranes for high performance wastewater microfiltration. Separation and Purification Technology 226:323-336

Aruchamy K, Mahto A, Nataraj S (2018) Electrospun nanofibers, nanocomposites and characterization of art: insight on establishing fibers as product. Nano-Structures \& Nano-Objects 16:45-58

Attari N, Hausler R (2020) Morphological investigation of cellulose acetate nanofibrous membranes. Proceedings of the 4rd International Conference of Recent Trends in Environmental Science and Engineering (RTESE'20)

Choi SS, Lee YS, Joo CW, Lee SG, Park JK, Han KS (2004) Electrospun pvdf nanofiber web as polymer electrolyte or separator. Electrochimica Acta 50(2-3):339-343

Del Río De Vicente JI (2021) Cellulose nanocrystals functionalized cellulose acetate electrospun membranes for adsorption and separation of nanosized particles

Etemadi H, Yegani R, Babaeipour V (2016) Study on the reinforcing effect of nanodiamond particles on the mechanical, thermal and antibacterial properties of cellulose acetate membranes. Diamond and Related Materials 69:166 176

Fischer S, Thümmler K, Volkert B, Hettrich K, Schmidt I, Fischer K (2008) Properties and applications of cellulose acetate. In: Macromolecular symposia, Wiley Online Library, vol 262, pp 89-96

Geng S, Wloch D, Herrera N, Oksman K (2020) Large-scale manufacturing of ultra-strong, strain-responsive poly (lactic acid)-based nanocomposites reinforced with cellulose nanocrystals. Composites Science and Technology p 108144

Ghasemi S, Behrooz R, Ghasemi I, Yassar RS, Long F (2018) Development of nanocellulose-reinforced pla nanocomposite by using maleated pla (plag-ma). Journal of Thermoplastic Composite Materials 31(8):1090-1101

Goetz LA, Naseri N, Nair SS, Karim Z, Mathew AP (2018) All cellulose electrospun water purification membranes nanotextured using cellulose nanocrystals. Cellulose 25(5):3011-3023

Golizadeh M, Karimi A, Gandomi-Ravandi S, Vossoughi M, Khafaji M, Joghataei MT, Faghihi F (2019) Evaluation of cellular attachment and 
proliferation on different surface charged functional cellulose electrospun nanofibers. Carbohydrate polymers 207:796-805

Hivechi A, Bahrami SH, Siegel RA, B Milan P, Amoupour M (2020) In vitro and in vivo studies of biaxially electrospun poly (caprolactone)/gelatin nanofibers, reinforced with cellulose nanocrystals, for wound healing applications. Cellulose pp 1-18

Janeca A, Rodrigues FS, Gonçalves MC, Faria M (2021) Novel cellulose acetate-based monophasic hybrid membranes for improved blood purification devices: Characterization under dynamic conditions. Membranes 11(11):825

Jiang L, Li K, Yang H, Liu X, Li W, Xu W, Deng B (2020) Improving mechanical properties of electrospun cellulose acetate nanofiber membranes by cellulose nanocrystals with and without polyvinylpyrrolidone. Cellulose $27(2): 955-967$

Jin K, Tang Y, Zhu X, Zhou Y (2020) Polylactic acid based biocomposite films reinforced with silanized nanocrystalline cellulose. International Journal of Biological Macromolecules 162:1109-1117

Jonoobi M, Harun J, Mathew AP, Oksman K (2010) Mechanical properties of cellulose nanofiber (cnf) reinforced polylactic acid (pla) prepared by twin screw extrusion. Composites Science and Technology 70(12):1742-1747

Lalia BS, Kochkodan V, Hashaikeh R, Hilal N (2013) A review on membrane fabrication: Structure, properties and performance relationship. Desalination 326:77-95

Lalia BS, Guillen E, Arafat HA, Hashaikeh R (2014) Nanocrystalline cellulose reinforced pvdf-hfp membranes for membrane distillation application. Desalination 332(1):134-141

Lee H, Nishino M, Sohn D, Lee JS, Kim IS (2018) Control of the morphology of cellulose acetate nanofibers via electrospinning. Cellulose 25(5):2829-2837

Levanic J, Šenk VP, Nadrah P, Poljanšek I, Oven P, Haapala A (2020) Analyzing tempo-oxidized cellulose fiber morphology: new insights into optimization of the oxidation process and nanocellulose dispersion quality. ACS Sustainable Chemistry \& Engineering 8(48):17752-17762

Liang Y, Cheng S, Zhao J, Zhang C, Sun S, Zhou N, Qiu Y, Zhang X (2013) Heat treatment of electrospun polyvinylidene fluoride fibrous membrane separators for rechargeable lithium-ion batteries. Journal of Power Sources 240:204-211

Liao Y, Loh CH, Tian M, Wang R, Fane AG (2018) Progress in electrospun polymeric nanofibrous membranes for water treatment: Fabrication, modification and applications. Progress in Polymer Science 77:69-94

Liu D, Sun X, Tian H, Maiti S, Ma Z (2013) Effects of cellulose nanofibrils on the structure and properties on pva nanocomposites. Cellulose 20(6):29812989

Liu H, Hsieh YL (2002) Ultrafine fibrous cellulose membranes from electrospinning of cellulose acetate. Journal of Polymer Science Part B: Polymer Physics 40(18):2119-2129 
Liu H, Tang C (2007) Electrospinning of cellulose acetate in solvent mixture n, n-dimethylacetamide (dmac)/acetone. Polymer journal 39(1):65-72

Ma H, Burger C, Hsiao BS, Chu B (2014) Fabrication and characterization of cellulose nanofiber based thin-film nanofibrous composite membranes. Journal of Membrane Science 454:272-282

Mokhena T, Jacobs N, Luyt A (2018) Nanofibrous alginate membrane coated with cellulose nanowhiskers for water purification. Cellulose 25(1):417-427

Ni X, Cheng W, Huan S, Wang D, Han G (2019) Electrospun cellulose nanocrystals/poly (methyl methacrylate) composite nanofibers: Morphology, thermal and mechanical properties. Carbohydrate polymers 206:29-37

Pan CY, Xu GR, Xu K, Zhao HL, Wu YQ, Su HC, Xu JM, Das R (2019) Electrospun nanofibrous membranes in membrane distillation: Recent developments and future perspectives. Separation and Purification Technology 221:44-63

Patiño-Masó J, Serra-Parareda F, Tarrés Q, Mutjé P, Espinach FX, DelgadoAguilar M (2019) Tempo-oxidized cellulose nanofibers: a potential bio-based superabsorbent for diaper production. Nanomaterials 9(9):1271

Salama A, Mohamed A, Aboamera NM, Osman T, Khattab A (2018) Characterization and mechanical properties of cellulose acetate/carbon nanotube composite nanofibers. Advances in Polymer Technology 37(7):2446-2451

Selatile MK, Ray SS, Ojijo V, Sadiku R (2018) Recent developments in polymeric electrospun nanofibrous membranes for seawater desalination. RSC advances 8(66):37915-37938

Sheng L, Jiang R, Zhu Y, Ji Y (2014) Electrospun cellulose nanocrystals/polycaprolactone nanocomposite fiber mats. Journal of Macromolecular Science, Part B 53(5):820-828

Stamatialis DF, Papenburg BJ, Gironés M, Saiful S, Bettahalli SN, Schmitmeier S, Wessling M (2008) Medical applications of membranes: Drug delivery, artificial organs and tissue engineering. Journal of Membrane Science 308(1-2):1-34

Suja P, Reshmi C, Sagitha P, Sujith A (2017) Electrospun nanofibrous membranes for water purification. Polymer reviews 57(3):467-504

Sun C, Boluk Y, Ayranci C (2015) Investigation of nanofiber nonwoven meshes produced by electrospinning of cellulose nanocrystal suspensions in cellulose acetate solutions. Cellulose 22(4):2457-2470

Tijing L, Woo Y, Yao M, Ren J, Shon H (2017) 1.16 electrospinning for membrane fabrication: strategies and applications. Comprehensive membrane science and engineering, 2nd edn Elsevier, Oxford pp 418-444

Vaidya R, Wilkins E (1994) Effect of interference on amperometric glucose biosensors with cellulose acetate membranes. Electroanalysis 6(8):677-682

Voisin H, Bergström L, Liu P, Mathew AP (2017) Nanocellulose-based materials for water purification. Nanomaterials $7(3): 57$

Wang X, Cheng W, Wang D, Ni X, Han G (2019) Electrospun polyvinylidene fluoride-based fibrous nanocomposite membranes reinforced by cellulose nanocrystals for efficient separation of water-in-oil emulsions. Journal of Membrane Science 575:71-79 
Wang Y, Ying Z, Xie W, Wu D (2020) Cellulose nanofibers reinforced biodegradable polyester blends: Ternary biocomposites with balanced mechanical properties. Carbohydrate Polymers 233:115845

Wsoo MA, Shahir S, Mohd Bohari SP, Nayan NHM, Razak SIA (2020) A review on the properties of electrospun cellulose acetate and its application in drug delivery systems: A new perspective. Carbohydrate Research 491:107978

Wu Y, Tang Q, Yang F, Xu L, Wang X, Zhang J (2019) Mechanical and thermal properties of rice straw cellulose nanofibrils-enhanced polyvinyl alcohol films using freezing-and-thawing cycle method. Cellulose 26(5):3193-3204

Yang J, Han CR, Duan JF, Ma MG, Zhang XM, Xu F, Sun RC, Xie XM (2012) Studies on the properties and formation mechanism of flexible nanocomposite hydrogels from cellulose nanocrystals and poly (acrylic acid). Journal of Materials Chemistry 22(42):22467-22480

You Y, Lee SW, Lee SJ, Park WH (2006) Thermal interfiber bonding of electrospun poly (l-lactic acid) nanofibers. Materials Letters 60(11):1331-1333

Zhou Z, Lin W, Wu XF (2016) Electrospinning ultrathin continuous cellulose acetate fibers for high-flux water filtration. Colloids and Surfaces A: Physicochemical and Engineering Aspects 494:21-29 